\title{
Production of Triply Heavy Baryons at LHC
}

\author{
Yu-Qi Chen and Su-Zhi Wu \\ Key Laboratory of Frontiers in Theoretical Physics, \\ Institute of Theoretical Physics, Chinese Academy of Sciences \\ Beijing 100190, P.R. China
}

\begin{abstract}
Triply heavy baryons are very interesting hadrons to be explored for they provide particular information about strong interactions, hadron structures, and weak decays of heavy baryons. We calculate the hadronic production cross sections of the $\Omega_{c c c}$ and the $\Omega_{c c b}$, which are dominated by the $g g$ fusion subprocesses containing 4362 and 1454 Feynman diagrams, respectively. A method for generating and calculating tree level Feynman diagrams automatically is developed to deal with complicated processes containing so many diagrams. Our results show that $10^{4}-10^{5}$ events of triply heavy baryons can be accumulated for $10 \mathrm{fb}^{-1}$ integrated luminosity at LHC. Signatures of the triply heavy baryons are pointed out, with emphasis on the decay modes $\Omega_{c c c} \rightarrow \Omega_{s s s}+3 \pi^{+}$ and $\Omega_{c c b} \rightarrow \Omega_{s s s}+3 \pi^{+}+\pi^{-}$. We conclude that it is quite promising to discover them at LHC.
\end{abstract}




\section{Introduction}

One interesting baryon to be discovered is the triply heavy baryon which is the color-singlet bound state consisting of three heavy $c$ or $b$ quarks by the strong interaction. Especially, exploring this baryon will be very useful for understanding the three body static potential. Since the mass $m$ of the heavy quark is so high, the relative motion of the quarks inside the triply heavy baryon is nonrelativistic and the typical velocity $v$ of the heavy quark in the rest frame of the baryon is small. The three heavy quarks are bound tightly and the typical size of this baryon being order of $1 / m v$ is smaller than that of the conventional baryons with light flavors. The mass of the baryon $\bar{M}$ is the sum of the three heavy quarks' masses and the binding energy which is of order $m v^{2}$. Similar with the heavy quarkonium system, in the limit of $v \ll 1$, there are three distinct energy scales involved in the triply heavy baryon system, i.e., the mass of heavy quark $m$, the three-momentum of the heavy quark $m v$, and the off-shell energy of the heavy quark $m v^{2}$. Nonrelativistic QCD effective theory (NRQCD) [1] can be used to describe the triply heavy baryon system in a simpler way by reducing the number of energy scales, where physics effects at energy scale $m$ are explicitly integrated out. Moreover, in the limits of both large $N_{c}$ and heavy quark mass, the mean field approximation can be applied to this system. Consequently, the wave function satisfies an ordinary differential equation [2]. Exploring this baryon certainly will enrich our knowledge about hadron structures and QCD interactions. In addition, the ground state can only decay via the weak interaction. Since the dynamics is relatively simpler, the triply heavy baryon provides very interesting samples to study the weak decays of hadrons. In particular, both the identical fermion effects and the larger recoil effects caused by the spectators can be studied well in theory. It may provide some insight for clarifying some long-standing puzzles in weak decays of heavy hadrons.

The masses, lifetimes, and other properties of these baryons have been studied over the past three decades [3, 4, 5, 6, 17, 8, 9, 10]. However, productions of these baryons are so difficult that they have not yet been discovered so far. They can be produced at high energy colliders by a direct production mechanism which can be described as follows. Triple heavy quark pairs are produced first at energy scale $m$ or higher, 
followed by the formation of the triply heavy baryons at energy scale $m v$. In the leading order approximation, three heavy quarks forming a color singlet state move together with smaller relative velocities $v$. Therefore, the production cross sections can be factored into short-distance coefficients and long-distance matrix elements. The short-distance coefficients describe the hard process effects of the triple heavy quark pairs production which can be calculated by perturbative QCD and expanded as a power series of $\alpha_{s}$ at energy scale $m$ or higher. The long-distance matrix elements describe the formation of the triply heavy baryon from point-like three heavy quarks with small relative velocity $v$. At hadron colliders, the triple heavy quark pairs can be produced via $g g$ fusion and quark-antiquark annihilation subprocess while at $e^{+} e^{-}$ colliders, it can be produced via $e^{+} e^{-}$annihilation process. The production cross section for the triply-charmed baryon in the $e^{+} e^{-}$collision has been calculated in Ref. [11] and the predicted production rate turns out to be very small. LHC, however, as a very high energy and high luminosity hadron collider, provides a very good chance to discover this sort of baryons due to very large heavy quark pairs production rates.

The authors in Refs. [12] and [13] calculated the hadronic production cross sections of the triply heavy baryons at LHC with their fragmentation functions. They derived fragmentation functions in the so-called diquark model of the heavy baryon and in perturbative calculation model, respectively. However, their obtained results do not represent the fragmentation functions for the production of triply heavy baryons predicted by QCD perturbation theory in the leading order.

In [12, the author calculated the fragmentation function in the diquark model of the triply heavy baryons by treating two of the three heavy quarks as a point-like diquark. This is inappropriate for the reason that two heavy quarks can be treated as a point-like diquark particle only when the momentum of the gluon emitted or absorbed from the diquark is much smaller than the inverse power of the typical size of the diquark which is order of $m v$. In the production process, this condition cannot be satisfied since the momenta of those gluons are order of $m$ or higher. Moreover, in his diquark description, the produced two free heavy antiquarks are forced to move in the same direction, which deviates from QCD description.

In Ref. [13], the authors calculated the fragmentation function using perturbation theory in Feynman gauge and including contributions only from two Feynman 
diagrams. As shown in Ref. [14, the fragmentation function can only be correctly calculated in axial gauge. Moreover, there are seven Feynman diagrams contributing to the leading order fragmentation function which are shown in Fig.1 in this paper.

Even with the correct QCD leading order fragmentation function, one cannot expect that it gives accurate description for the triply heavy baryons at small $P_{T}$. In Ref. [15], the authors did a comparative studied for the hadronic $B_{c}$ production by full QCD and by the fragmentation approximation. Their results show that the fragmentation approximation is valid only when the transverse momentum of the heavy hadron, $P_{T}$, is much larger than its mass $\bar{M}$. However, in hadron collisions, the production cross sections are dominated in the smaller $P_{T}$ region, typically comparable with $\bar{M}$. Thus one can not expect that the fragmentation approximation gives accurate results. Instead, calculations of the production cross sections by full perturbative QCD are necessary and expected to give reliable predictions.

In this paper, we study the production rates of the triply heavy baryons at LHC. We first calculate the short-distance coefficients in the leading order of $\alpha_{s}^{6}$ corresponding to the tree level contributions. Even at the tree level, there are a number of Feynman diagrams that need to be calculated and it turns out to be hard work. For instance, there are as many as 4362 Feynman diagrams for $g g \rightarrow \Omega_{c c c}+\bar{c} \bar{c} \bar{c}$ and as many as 1454 Feynman diagrams for $g g \rightarrow \Omega_{c c b}+\bar{c} \bar{c} \bar{b}$. It is impossible to carry out the calculations by conventional calculation methods for cross sections.

For a simpler production process $e^{+} e^{-} \rightarrow \Omega_{c c c}+\bar{c} \bar{c} \bar{c}$, to simplify the calculation, the authors in Ref. [11] took an approximation ignoring the heavy quark mass terms in the numerator of the quark propagator. The approximation significantly simplifies the calculations. However, as pointed out in Ref. [16], the approximation will lead to quite large errors with the same order contributions.

To overcome the difficulty meeting here, we propose a method to evaluate Feynman diagrams by generating the diagrams automatically and use a direct amplitude calculation of the Feynman diagrams. The amplitudes are classified as a set of gauge invariant subsets in terms of independent color structures. Consequently, the code for numerical calculations is written in a significant compact form and numerical calculations of the amplitudes can be carried out. To ensure the correctness of the calculations, we verify various gauge invariance of the amplitude of each subset to 
examine the code.

Taking the long-distance matrix elements given in [6], the cross sections of the subprocesses can be calculated. Incorporating with the parton model, we calculate the production cross sections of the triply heavy baryons at LHC. Our results show that $10^{4}-10^{5}$ events of triply heavy baryons $\Omega_{c c c}$ and $\Omega_{c c b}$ can be accumulated for $10 \mathrm{fb}^{-1}$ integrated luminosity at LHC. We also present $P_{T^{-}}$distributions and rapidity distributions with various parameters. In this paper, we consider only the ground states with all orbital angular momenta vanishing. We also point out the signatures to reconstruct the triply heavy baryons, with $\Omega_{c c c} \rightarrow \Omega_{s s s}+3 \pi^{+}$and $\Omega_{c c b} \rightarrow \Omega_{s s s}+$ $3 \pi^{+}+\pi^{-}$being emphasized. We conclude that it is quite promising to discover them at LHC.

The rest of the paper is organized as follows. In Sec 2, we present the calculation of the subprocesses of $g g(q \bar{q}) \rightarrow \Omega_{Q_{1} Q_{2} Q_{3}} \bar{Q}_{1} \bar{Q}_{2} \bar{Q}_{3}$. In Sec. 3, numerical results of the total cross sections and differential distributions are presented. Sec. 4 contributes to the signatures of these baryons and conclusions.

\section{$2 \quad$ Subprocess of $g g(q \bar{q}) \rightarrow \Omega_{Q_{1} Q_{2} Q_{3}} \bar{Q}_{1} \bar{Q}_{2} \bar{Q}_{3}$}

In this section, we calculate the cross sections of the production subprocesses of $\Omega_{Q_{1} Q_{2} Q_{3}}$ at LHC in the leading order. There are a number of Feynman diagrams contributing to the short distance coefficients. We carry out the calculations by taking a strategy to generating the diagrams automatically and using a direct amplitude calculations of the Feynman diagrams. Consequently, the code for numerical calculations is written in a very compact form. We present the calculations in detail below.

\subsection{Production mechanism of the triply heavy baryon}

The triply heavy baryon is the color-singlet bound state of three heavy quarks by the strong interaction. However, since the mass $m$ of the heavy quark is much larger than $\Lambda_{Q C D}$, the relative motion of the quarks inside the triply heavy baryon is nonrelativistic and the typical velocity $v$ of the heavy quark in the rest frame of the baryon is small. The three heavy quarks are bound tightly and the typical size of this baryon being of order $1 / m v$ is smaller than that of the conventional baryons 
with light flavors which is of order $1 / \Lambda_{Q C D}$. The mass of the state is the sum of the three heavy quark masses and the binding energy which is of order $m v^{2}$. In the limit of $v \ll 1$, there are three distinct energy scales involved in the triply heavy system, i.e., the mass of the heavy quark $m$, the three-momentum of the heavy quark $m v$, and the off-shell energy of the heavy quark $m v^{2}$. NRQCD [1] can be used to describe the system conveniently, where physics effects at energy scale $m$ are explicitly integrated out.

The triply heavy baryon, $\Omega_{Q_{1} Q_{2} Q_{3}}$, being in a color singlet state implies that its color wavefunction must be of the form $\frac{1}{\sqrt{6}} \varepsilon^{\xi_{1} \xi_{2} \xi_{3}} Q_{1 \xi_{1}} Q_{2 \xi_{2}} Q_{3 \xi_{3}}$, where $\xi_{i}(i=1,2,3)$ are the color indices of the heavy quark $Q_{i}$. The heavy quarks may be either the $c$ quark or the $b$ quark. The triply heavy baryons can be classified as two classes in terms of containing two or three identical heavy quarks. For those orbital angular momentum ground states, the exchange antisymmetry of the identical fermion implies that the triply heavy baryons with three identical heavy quarks can only be the spinsymmetrical states and only spin $\frac{3}{2}$ states are allowed, while the triply heavy baryons with double identical heavy quarks can be either the spin-symmetrical state with spin $3 / 2$ or the other one with spin $1 / 2$. We take $\Omega_{c c c}, \Omega_{c c b}^{*}$ and $\Omega_{c c b}$ to denote the triply heavy baryons consisting of the $c c c$ quarks with spin $3 / 2$, the $c c b$ quarks with spin $3 / 2$ and spin $1 / 2$, respectively.

The mass of the triply heavy baryon is so high that it is very difficult to produce at normal high energy machines. At LHC, they can be produced by a direct production mechanism in which triple heavy quark pairs are produced first at energy scale $m$ or higher by $g g$ fusion or $q \bar{q}$ annihilation subprocesses, followed by the formation of the triply heavy baryons by combining the color-singlet triple heavy quarks moving in the same directions with small relative velocities at energy scale $m v$. In the heavy quark limit, $v \ll 1$, the production cross sections can be factored into the product of short-distance coefficients and the long-distance matrix elements. The short-distance coefficients describe the hard process effects of the triple heavy quark pairs production. They can be calculated by perturbative QCD and be expanded as a power series of $\alpha_{s}$ at energy scale $m$ or higher. The long-distance matrix elements describe the formation of the triply heavy baryons from point-like three heavy quarks with small relative velocities. In this paper, we consider only the contributions arising from the leading 
order short distance coefficient and the leading order long-distance matrix element. There is only single long-distance matrix element. The factorization then holds at amplitude level which we illustrate below. Given the leading Fock state description presented in Appendix 6.1, by the standard perturbation theory, the amplitude of the subprocess $g g \quad(q \bar{q}) \rightarrow \Omega_{Q_{1} Q_{2} Q_{3}} \bar{Q}_{1} \bar{Q}_{2} \bar{Q}_{3}$ reads:

$$
\begin{aligned}
A\left(g g(q \bar{q}) \rightarrow \Omega_{Q_{1} Q_{2} Q_{3}} \bar{Q}_{1} \bar{Q}_{2} \bar{Q}_{3}\right) & =\int \frac{d^{3} V_{1}}{(2 \pi)^{3}} \frac{d^{3} V_{2}}{(2 \pi)^{3}} \frac{\sqrt{2 \bar{M}}}{\sqrt{8 m_{1} m_{2} m_{3}}} \\
\frac{\psi\left(\vec{V}_{1}, \vec{V}_{2}\right)}{\sqrt{d !}} & \times M\left(g g(q \bar{q}) \rightarrow\left(Q_{1} Q_{2} Q_{3}\right)_{1}^{\left(s, s_{Z}\right)} \bar{Q}_{1} \bar{Q}_{2} \bar{Q}_{3}\right),
\end{aligned}
$$

where $\bar{M}\left(\bar{M}=m_{1}+m_{2}+m_{3}\right)$ is the mass of the baryon $\Omega_{Q_{1} Q_{2} Q_{3}}, m_{i}$ is the mass of $Q_{i}(\mathrm{i}=1,2,3), \psi\left(\vec{V}_{1}, \vec{V}_{2}\right)$ is the wave function of the baryon $\Omega_{Q_{1} Q_{2} Q_{3}}$ in momentum space; $\vec{V}_{1}, \vec{V}_{2}$ and $-\left(\vec{V}_{1}+\vec{V}_{2}\right)$ are the relative momenta among the three quarks in this baryon; $d=2,3$ is the number of the identical heavy quarks in the baryon arising from the identical fermion exchange antisymmetry and $\left(Q_{1} Q_{2} Q_{3}\right)_{1}^{\left(s, s_{Z}\right)}$ denotes the color-singlet state consisting of three heavy quarks with spin quantum number $s$ and its third component $s_{Z}$. A heavy quark with a large mass moves in the triply heavy baryon with a small velocity $v$ (in the baryon's rest frame). $M(g g(q \bar{q})$ fusion) depending on $\vec{V}_{1}$ and $\vec{V}_{2}$ describes the hard amplitude for producing the triple heavy quark pairs. The typical momenta are of order $m$ or higher. Thus in the leading order (expansion in $v$ ) approximation, the $\vec{V}_{1}, \vec{V}_{2}$ dependence in the matrix element $M\left(g g(q \bar{q})\right.$ fusion) can be neglected. The integration over variables $\vec{V}_{1}$ and $\vec{V}_{2}$ can be carried out. It follows that from equation (11):

$$
\begin{aligned}
& A\left(g g(q \bar{q}) \rightarrow \Omega_{Q_{1} Q_{2} Q_{3}} \bar{Q}_{1} \bar{Q}_{2} \bar{Q}_{3}\right)= \\
& \frac{\sqrt{2 \bar{M}}}{\sqrt{8 m_{1} m_{2} m_{3}}} \frac{\Psi(0,0)}{\sqrt{d !}} M\left(g g(q \bar{q}) \rightarrow\left(Q_{1} Q_{2} Q_{3}\right)_{1}^{\left(s, s_{Z}\right)} \bar{Q}_{1} \bar{Q}_{2} \bar{Q}_{3}\right)
\end{aligned}
$$

where $\Psi(0,0)$ is value of the space wave function of the baryon $\Omega_{Q_{1} Q_{2} Q_{3}}$ when the three quarks are all at the origin which describes the long-distance effects that happen at energy scale $m v$. In this way, the long-distance and short-distance effects are separated at the amplitude level in the leading order approximation. Our task now is to calculate the short-distance amplitude $M\left(g g(q \bar{q}) \rightarrow\left(Q_{1} Q_{2} Q_{3}\right)_{1}^{\left(s, s_{Z}\right)} \bar{Q}_{1} \bar{Q}_{2} \bar{Q}_{3}\right)$, which describes triple heavy-quark pair production with the triple quarks in a color-singlet 

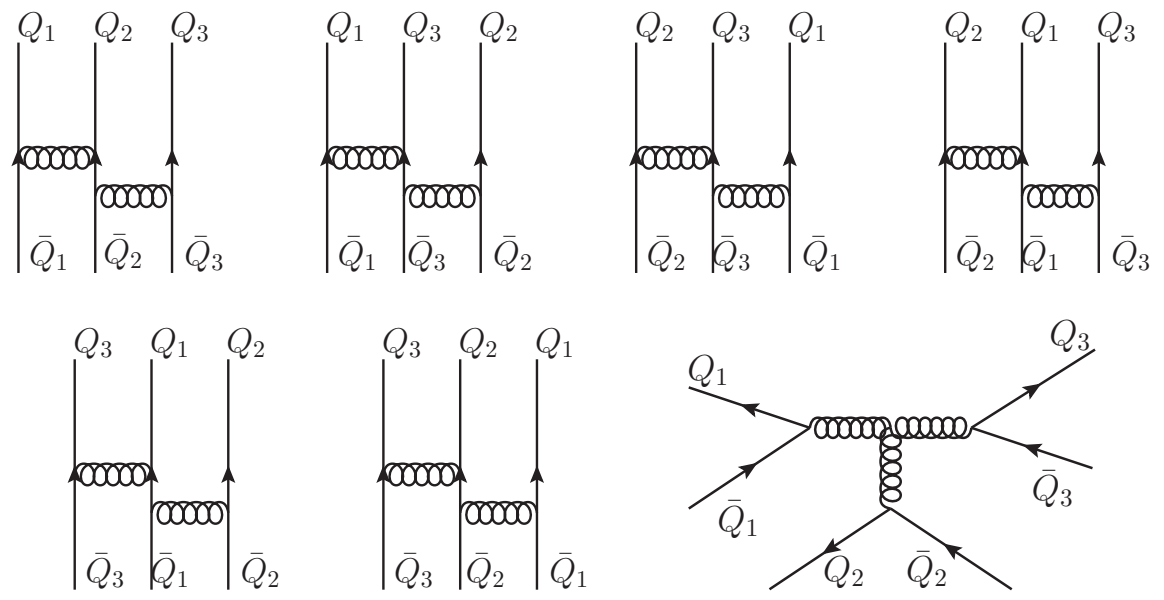

Figure 1: Seven basic diagrams for generating the Feynman diagrams of the triply heavy baryon production subprocess with different heavy flavors.

and point-like $\left(Q_{1} Q_{2} Q_{3}\right)_{1}^{\left(s, s_{Z}\right)}$ state. In calculating the amplitude, the momentum of each quark in the $\left(Q_{1} Q_{2} Q_{3}\right)_{1}^{\left(s, s_{Z}\right)}$ state satisfies $P_{Q_{1}}: P_{Q_{2}}: P_{Q_{3}}=m_{1}: m_{2}: m_{3}$.

In this paper, we consider only the triply heavy baryons production of the $\Omega_{c c c}$, $\Omega_{c c b}^{*}$, and $\Omega_{c c b}$ states. Since the production rates of other ones such as $\Omega_{b b b}, \Omega_{c b b}^{*}$, and $\Omega_{c b b}$ are small, they are not included in this paper.

\section{$2.2 \quad$ Feynman diagram generating}

Since there are so many Feynman diagrams responsible for the amplitude of the subprocess, to carry out the calculation, it is crucial to generate the Feynman diagrams automatically. We now propose a method to realize it by starting from the triply heavy baryons production via $g g$ fusion subprocess. Notice that removing the external gluon lines and the corresponding interaction vertex for $g g$ fusion subprocess, all Feynman diagrams reduce to seven basic diagrams as shown in Fig 1) 1assuming three heavy quarks with different heavy flavors. They are nothing but the diagrams connecting three heavy quark lines in various ways. It means that all Feynman diagrams for $g g$ fusion subprocess can be obtained by inserting two external gluon lines into all possible positions of the 7 basic diagrams shown in Fig.1.

For the production process of $\Omega_{c c c}$, the exchange anti-symmetry of the identical fermions means that the number of the basic Feynman diagrams is $7 \times 3$ ! $=42$, while 
for the production process of $\Omega_{c c b}$, it means that the number of the basic Feynman diagrams is $7 \times 2 !=14$.

We now show how to generate all Feynman diagrams for the triple heavy quark pairs production subprocess via $g g$ fusion. Notice that every diagram contains at most single quadruple-gluon vertex. For convenience, all diagrams are divided into two classes in terms of whether they contain the quadruple-gluon vertex or not.

We first generate Feynman diagrams without quadruple-gluon vertex by inserting the external gluon lines in between the gluon or quark lines of the basic diagrams one by one. There are 3 internal and 6 external lines for each of the basic Feynman diagram. The first external gluon can be inserted in between one of these 9 lines. Then there are 4 internal and 7 external lines. And the second gluon $g$ can be inserted in between one of these 11 lines. Therefore, for the production process of the triply heavy baryon with three different flavors, the number of the diagrams without quadruple-gluon vertex is $7 \times 9 \times 11=693$.

We then generate the Feynman diagrams with one quadruple-gluon vertex. They are generated either from the last diagram in Fig,1, where one gluon attaches to the triple-gluon vertex while the other one inserts into all other possible lines, or from all 7 basic diagrams with the double external gluon lines inserting into the same point of any gluon line. These two ways generate 19 and 15 diagrams with quadruple-gluon vertex, respectively. Thus totally one has $693+34=727$ Feynman diagrams.

For the $\Omega_{c c b}\left(\Omega_{c c b}^{*}\right)$ and $\Omega_{c c c}$ production via the $g g$ fusion subprocesses, there are $727 \times 2 !=1454$ and $727 \times 3 !=4362$ Feynman diagrams, respectively. In this way, we generate all Feynman diagrams responsible for the tree level amplitude of the triply heavy baryon production via the $g g$ fusion subprocesses.

In a similar way, we now can generate all Feynman diagrams for the triple heavy quark pairs production via the quark-antiquark annihilation subprocesses. In the production subprocess of $q \bar{q}$ annihilating to three quark pairs with different flavors, Feynman diagrams can be classified as three types in terms of the number of gluon lines attached to the light quark lines. For the first type diagrams with one gluon attached to the light quark line, there are $7 \times 9=63$ Feynman diagrams without quadruple-gluon vertex and 1 Feynman diagram with one quadruple-gluon vertex. For the second type diagrams with double gluon lines attached to the light quark 
lines, the Feynman diagrams can be generated by breaking up one of the gluon lines in the 7 basic diagrams and attaching to the light quark line. For this type, totally there are $2 ! \times 15=30$ Feynman diagrams. For the third type diagrams with triple gluon lines attached to the light quark lines, all gluon lines between heavy quarks are removed, and each of three gluon lines are attached to each of three heavy quark lines. Thus for this type, there are $3 ! \times 1=6$ diagrams. Adding the three types of Feynman diagrams together, totally there are $64+30+6=100$ Feynman diagrams for the process of $q \bar{q}$ annihilation to three heavy quark pairs with different flavors. Thus for the $\Omega_{c c b}\left(\Omega_{c c b}^{*}\right)$ and $\Omega_{c c c}$ production subprocesses via the quark-antiquark annihilation, there are $100 \times 2 !=200$ Feynman diagrams and $100 \times 3 !=600$ ones, respectively.

\subsection{Color factors of the amplitude}

The large number of Feynman diagrams makes the calculation very complicated. To carry out the calculations we first need to account for the color factors. The amplitude can be classified into a set of gauge invariant subsets in terms of variant independent color factors. Denote $\xi_{i}=1,2,3, \chi_{i}=1,2,3(i=1,2,3), a, b=$ $1,2, \cdots, 8$, as the color indices of three heavy quarks, three anti-heavy quarks, and two gluons, respectively. For the $g g$ fusion production subprocess, there are totally 12 independent color factors $C_{k}(k=1,2, \cdots, 12)$ listed as follows:

$$
\begin{array}{rlrl}
C_{1} & =\frac{\varepsilon^{\xi_{1} \xi_{2} \xi_{3}}}{\sqrt{6}}\left(\lambda^{a} \lambda^{b}\right)_{\xi_{1} \chi_{2}} \delta_{\xi_{2} \chi_{1}} \delta_{\xi_{3} \chi_{3}}, & C_{2}=\frac{\varepsilon^{\xi_{1} \xi_{2} \xi_{3}}}{\sqrt{6}}\left(\lambda^{b} \lambda^{a}\right)_{\xi_{1} \chi_{2}} \delta_{\xi_{2} \chi_{1}} \delta_{\xi_{3} \chi_{3}}, \\
C_{3}=\frac{\varepsilon^{\xi_{1} \xi_{2} \xi_{3}}}{\sqrt{6}}\left(\lambda^{a} \lambda^{b}\right)_{\xi_{1} \chi_{1}} \delta_{\xi_{2} \chi_{2}} \delta_{\xi_{3} \chi_{3}}, & C_{4}=\frac{\varepsilon^{\xi_{1} \xi_{2} \xi_{3}}}{\sqrt{6}}\left(\lambda^{b} \lambda^{a}\right)_{\xi_{1} \chi_{1}} \delta_{\xi_{2} \chi_{2}} \delta_{\xi_{3} \chi_{3}}, \\
C_{5}=\frac{\varepsilon^{\xi_{1} \xi_{2} \xi_{3}}}{\sqrt{6}}\left(\lambda^{a} \lambda^{b}\right)_{\xi_{1} \chi_{3}} \delta_{\xi_{2} \chi_{1}} \delta_{\xi_{3} \chi_{2}}, & C_{6}=\frac{\varepsilon^{\xi_{1} \xi_{2} \xi_{3}}}{\sqrt{6}}\left(\lambda^{b} \lambda^{a}\right)_{\xi_{1} \chi_{3}} \delta_{\xi_{2} \chi_{1}} \delta_{\xi_{3} \chi_{2}}, \\
C_{7}=\frac{\varepsilon^{\xi_{1} \xi_{2} \xi_{3}}}{\sqrt{6}}\left(\lambda^{a}\right)_{\xi_{1} \chi_{1}}\left(\lambda^{b}\right)_{\xi_{2} \chi_{2}} \delta_{\xi_{3} \chi_{3}}, & C_{8}=\frac{\varepsilon^{\xi_{1} \xi_{2} \xi_{3}}}{\sqrt{6}}\left(\lambda^{a}\right)_{\xi_{1} \chi_{2}}\left(\lambda^{b}\right)_{\xi_{2} \chi_{1}} \delta_{\xi_{3} \chi_{3}} \\
C_{9}=\frac{\varepsilon^{\xi_{1} \xi_{2} \xi_{3}}}{\sqrt{6}}\left(\lambda^{a}\right)_{\xi_{1} \chi_{2}}\left(\lambda^{b}\right)_{\xi_{2} \chi_{3}} \delta_{\xi_{3} \chi_{1}}, & C_{10}=\frac{\varepsilon^{\xi_{1} \xi_{2} \xi_{3}}}{\sqrt{6}}\left(\lambda^{a}\right)_{\xi_{1} \chi_{3}}\left(\lambda^{b}\right)_{\xi_{2} \chi_{2}} \delta_{\xi_{3} \chi_{1}} \\
C_{11}=\frac{\varepsilon^{\xi_{1} \xi_{2} \xi_{3}}}{\sqrt{6}}\left(\lambda^{a}\right)_{\xi_{1} \chi_{3}}\left(\lambda^{b}\right)_{\xi_{2} \chi_{1}} \delta_{\xi_{3} \chi_{2}}, & C_{12}=\frac{\varepsilon^{\xi_{1} \xi_{2} \xi_{3}}}{\sqrt{6}}\left(\lambda^{a}\right)_{\xi_{1} \chi_{1}}\left(\lambda^{b}\right)_{\xi_{2} \chi_{3}} \delta_{\xi_{3} \chi_{2}} .
\end{array}
$$

The color factors given in Eq. (3) can be used as the independent bases of the color wave function of the amplitude of the $g g$ fusion subprocess. With these color factors, 
the amplitude of each diagram can be expressed as the sum of the products of the numerical values and those color factors. It yields:

$$
\mathcal{M}=d ! \sum_{k=1}^{12} C_{k} \Gamma_{k}
$$

where $\Gamma_{k}$ is the amplitude of the basic Feynman diagrams related to the color-factor $C_{k}$.

Denote $\varsigma_{1}=1,2,3$ and $\varsigma_{2}=1,2,3$ the color indices of $q$ and $\bar{q}$ for $q \bar{q}$ annihilation subprocess. There are totally 4 independent color factors $D_{k}(k=1,2,3,4)$ for the triple heavy quark pair production via $q \bar{q}$ annihilation subprocess listed as follows:

$$
\begin{aligned}
& D_{1}=\frac{\varepsilon^{\xi_{1} \xi_{2} \xi_{3}}}{\sqrt{6}} \delta_{\varsigma_{1} \chi_{1}} \delta_{\varsigma_{2} \xi_{1}} \delta_{\xi_{2} \chi_{2}} \delta_{\xi_{3} \chi_{3}} \\
& D_{2}=\frac{\varepsilon^{\xi_{1} \xi_{2} \xi_{3}}}{\sqrt{6}} \delta_{\varsigma_{1} \chi_{2}} \delta_{\varsigma_{2} \xi_{1}} \delta_{\xi_{2} \chi_{3}} \delta_{\xi_{3} \chi_{1}}, \\
& D_{3}=\frac{\varepsilon^{\xi_{1} \xi_{2} \xi_{3}}}{\sqrt{6}} \delta_{\varsigma_{1} \chi_{3}} \delta_{\varsigma_{2} \xi_{1}} \delta_{\xi_{2} \chi_{1}} \delta_{\xi_{3} \chi_{2}}, \\
& D_{4}=\frac{\varepsilon^{\xi_{1} \xi_{2} \xi_{3}}}{\sqrt{6}} \delta_{\varsigma_{1} \varsigma_{2}} \delta_{\xi_{1} \chi_{1}} \delta_{\xi_{2} \chi_{2}} \delta_{\xi_{3} \chi_{3}} .
\end{aligned}
$$

The color factors given in Eq. (5) can be used as the independent bases of the color wave function of the amplitude of the $q \bar{q}$ annihilation subprocess. With these color factors, the amplitude of each diagram can be expressed as the sum of the products of the numerical values and those color factors. It follows that:

$$
\mathcal{M}=d ! \sum_{k=1}^{4} D_{k} \Gamma_{k}^{\prime}
$$

where $\Gamma_{k}^{\prime}$ is the amplitude of the basic Feynman diagrams related to the color-factor $D_{k}$.

For those diagrams without the quadruple-gluon vertex, the amplitude of each diagram can be expressed as single product of a numerical value and a color factor, each of which can be decomposed as the sum of the color factors listed above. For those diagrams with the quadruple-gluon vertex, the amplitude of each diagram can be expressed as a sum of three products of numerical values and color factors, again, each of which can be decomposed as the sum of the color factors listed above. 


\subsection{Simplification by identical Fermionic symmetry}

As discussed in Sec. 2.2, the permutation of the identical heavy quarks increases the number of Feynman diagrams by a factor 3 ! and 2!, for the production of colorsinglet triple $c$ quark state and double $c$ quarks plus single $b$ quark one, respectively. Consequently, there are 4362 and 1454 Feynman diagrams that need to be calculated for the amplitudes of $\Omega_{c c c}$ and $\Omega_{c c b}\left(\Omega_{c c b}^{*}\right)$, respectively, via $g g$ fusion subprocesses. For the quark-antiquark annihilation subprocesses, the number of the Feynman diagrams are 200 and 600 for the production amplitudes of the $\Omega_{c c c}$ and the $\Omega_{c c b}\left(\Omega_{c c b}^{*}\right)$, respectively. Notice that the momenta of the identical heavy quarks in triply heavy baryons can be taken to be equal in calculating the amplitudes. This feature together with the identical fermion exchanging symmetry can be used to simplify the calculation. In fact, the contributions to the amplitude from those increased diagrams by each permutation of the identical heavy quarks are equal to the original diagrams. Thus one needs to calculate only those 727 diagrams generated via 7 basic diagrams for the $g g$ fusion subprocesses, and those 100 diagrams for the $q \bar{q}$ annihilation subprocesses. Thus the calculations of the amplitudes can be simplified significantly. We emphasize here that it is available only for the amplitude of the leading order matrix elements.

\subsection{Automatic amplitude calculation}

With the simplification in the last subsection, the number of the Feynman diagrams that need to be calculated is reduced to 727 and 100 for the production of the triply heavy baryons via the gluon-gluon fusion subprocess and the quark-antiquark annihilation subprocess, respectively. These are still very large numbers for doing numerical calculations. It would be quite lengthy and very tedious to code for numerical calculations if they are evaluated and expressed by the conventional method. Instead we develop a method to calculate Feynman diagrams closely related to the method for generating Feynman diagrams described in Sec. 2.2, incorporating a direct, numerical amplitude calculation method given in Ref. [17] as described as follows.

Given the quantum numbers and momenta of the initial and final states in the amplitude, all momenta of the internal lines are fixed in a specific tree level Feynman diagram. The propagators and vertices in the Feynman diagram are described by 
certain matrix or tensor while the spinor wavefunctions of the initial state and final state are described by the line and raw vectors, respectively 1 Then the calculation of the amplitude of the corresponding Feynman diagram is nothing but the product of the matrices to a numerical number against Fermion lines. The method turns out to be efficient in numerical calculation of tree level amplitude. However, for subprocesses with too many Feynman diagrams like the triply heavy baryons production one, it is still difficult to write down the amplitude of all diagrams one by one.

To overcome this issue, we develop an automatic calculation method, closely following the method for generating Feynman diagrams described in Sec. 2.2. Notice that the Feynman diagrams differ from each other by the positions and the types of propagators and vertices. Each type is assigned to a fix number. A Feynman diagram is then represented by a table consisting of a set of ordered numbers, each of which corresponds to the type of the propagator or vertex in the diagram. With the quantum numbers and the momenta of each propagator or vertex, the Feynman diagram can be calculated by the multiplication of the matrices or tensors controlled by the number in the table. In this way, the program code for calculating the amplitudes can be written in a compact form and contributions from all diagrams can be calculated conveniently. In numerical simulation of each event, we assign the momenta, helicities of the light quarks, the heavy antiquarks, and the heavy baryon, and the polarization vectors of gluons randomly, assuming that they meet a set of conservation laws. We then use Vegas to simulate the events and carry out the phase space integrals.

To ensure the correctness of the program code, we examine various gauge invariance of the amplitude by Ward-Takahashi identities of the $g g$ fusion subprocess. Namely, substituting one of two polarization vectors of gluons into its momentum, the total amplitude vanishes. It follows from Eq. (4) that each amplitude $\Gamma_{k}(k=1,2, \ldots, 12)$ vanishes under the substitution. To have additional test, in a similar way, we can also examine the triply heavy baryons production processes via double photons and $\gamma g$ fusion subprocess, in which the amplitudes are expressed as the sum of different gauge independent subsets. Similarly, we can also have various test for the program code for the subprocess of the $q \bar{q}$ annihilation.

\footnotetext{
${ }^{1}$ In Appendix 6.2, we list the 4-dimension vectors describing the spinors of the quark and antiquark, and the polarization vectors of the gluons.
} 


\subsection{The cross sections of the subprocess}

We have calculated the amplitude of the subprocesses $g g(q \bar{q})$ to triple heavy quark pairs above. When the amplitude is expressed as Eq. (41), using the formula given in Appendix 6.3, the squared amplitude for the $g g$ fusion subprocess reads:

$$
\begin{aligned}
\sum_{a, b, \chi_{i}}|\mathcal{M}|^{2} & =(d !)^{2}\left(\frac{64}{9} \sum_{k=1}^{12}\left|\Gamma_{k}\right|^{2}+2 R e\left[-\frac{8}{9} \sum_{k=1,3,5} \Gamma_{k} \Gamma_{k+1}^{*}\right.\right. \\
& +\frac{20}{9}\left(\Gamma_{1} \Gamma_{3}^{*}+\Gamma_{1} \Gamma_{5}^{*}+\Gamma_{2} \Gamma_{4}^{*}+\Gamma_{2} \Gamma_{6}^{*}-\Gamma_{3} \Gamma_{5}^{*}-\Gamma_{4} \Gamma_{6}^{*}+\Gamma_{7} \Gamma_{8}^{*}+\Gamma_{9} \Gamma_{10}^{*}\right. \\
& \left.+\Gamma_{11} \Gamma_{12}^{*}\right)+\frac{32}{9}\left(\Gamma_{1} \Gamma_{7}^{*}-\Gamma_{1} \Gamma_{10}^{*}-\Gamma_{2} \Gamma_{8}^{*}+\Gamma_{2} \Gamma_{9}^{*}+\Gamma_{3} \Gamma_{8}^{*}-\Gamma_{3} \Gamma_{11}^{*}-\Gamma_{4} \Gamma_{7}^{*}\right. \\
& +\Gamma_{4} \Gamma_{12}^{*}-\Gamma_{5} \Gamma_{9}^{*}+\Gamma_{5} \Gamma_{12}^{*}+\Gamma_{6} \Gamma_{10}^{*}-\Gamma_{6} \Gamma_{11}^{*}+\Gamma_{7} \Gamma_{10}^{*}+\Gamma_{7} \Gamma_{12}^{*}+\Gamma_{8} \Gamma_{9}^{*} \\
& \left.+\Gamma_{8} \Gamma_{11}^{*}+\Gamma_{9} \Gamma_{12}^{*}+\Gamma_{10} \Gamma_{11}^{*}\right)-\frac{16}{9}\left(\Gamma_{1} \Gamma_{4}^{*}+\Gamma_{1} \Gamma_{6}^{*}+\Gamma_{1} \Gamma_{11}^{*}-\Gamma_{1} \Gamma_{12}^{*}\right. \\
& +\Gamma_{2} \Gamma_{3}^{*}+\Gamma_{2} \Gamma_{5}^{*}+\Gamma_{2} \Gamma_{11}^{*}-\Gamma_{2} \Gamma_{12}^{*}-\Gamma_{3} \Gamma_{6}^{*}-\Gamma_{3} \Gamma_{9}^{*}+\Gamma_{3} \Gamma_{10}^{*}-\Gamma_{4} \Gamma_{5}^{*} \\
& -\Gamma_{4} \Gamma_{9}^{*}+\Gamma_{4} \Gamma_{10}^{*}-\Gamma_{5} \Gamma_{7}^{*}+\Gamma_{5} \Gamma_{8}^{*}-\Gamma_{6} \Gamma_{7}^{*}+\Gamma_{6} \Gamma_{8}^{*}-\Gamma_{7} \Gamma_{9}^{*}-\Gamma_{7} \Gamma_{11}^{*} \\
& \left.-\Gamma_{8} \Gamma_{10}^{*}-\Gamma_{8} \Gamma_{12}^{*}-\Gamma_{9} \Gamma_{11}^{*}-\Gamma_{10} \Gamma_{12}^{*}\right)+\frac{4}{9}\left(\Gamma_{1} \Gamma_{8}^{*}-\Gamma_{1} \Gamma_{9}^{*}-\Gamma_{2} \Gamma_{7}^{*}+\Gamma_{2} \Gamma_{10}^{*}\right. \\
& \left.\left.\left.+\Gamma_{3} \Gamma_{7}^{*}-\Gamma_{3} \Gamma_{12}^{*}-\Gamma_{4} \Gamma_{8}^{*}+\Gamma_{4} \Gamma_{11}^{*}-\Gamma_{5} \Gamma_{10}^{*}+\Gamma_{5} \Gamma_{11}^{*}+\Gamma_{6} \Gamma_{9}^{*}-\Gamma_{6} \Gamma_{12}^{*}\right)\right]\right)
\end{aligned}
$$

where, Re means taking the real part of the number, and $\sum_{a, b, \chi_{i}}$ means the summation of the color indices of the initial gluons and terminal heavy antiquarks.

Similarly, with the amplitude given in Eq. ([6) , the squared amplitude for the $q \bar{q}$ annihilation subprocess reads:

$$
\begin{aligned}
\sum_{\zeta_{1}, \zeta_{2}, \chi_{i}}|\mathcal{M}|^{2} & =(d !)^{2}\left(3 \sum_{i=1}^{4} \Gamma_{i} \Gamma_{i}^{*}-2 \cdot \operatorname{Re}\left(\Gamma_{1}^{\prime} \Gamma_{2}^{\prime *}+\Gamma_{1}^{\prime} \Gamma_{3}^{*}+\Gamma_{2}^{\prime} \Gamma_{3}^{* *}\right)\right. \\
& \left.+2 \cdot \operatorname{Re}\left(\Gamma_{1}^{\prime} \Gamma_{4}^{\prime *}+\Gamma_{2}^{\prime} \Gamma_{4}^{\prime *}+\Gamma_{3}^{\prime} \Gamma_{4}^{\prime *}\right)\right)
\end{aligned}
$$

The differential cross section of the subprocess is then given by:

$$
\begin{aligned}
& d \hat{\sigma}\left(g g \quad(q \bar{q}) \rightarrow \Omega_{Q_{1} Q_{2} Q_{3}} \bar{Q}_{1} \bar{Q}_{2} \bar{Q}_{3}\right) \\
= & \frac{1}{d !} d \Pi_{4} \frac{1}{(2 \pi)^{8} 2 \hat{s}} \frac{8}{N} \sum_{s_{Z}} \sum_{a, b, \varsigma_{1}, \varsigma_{2}, \chi_{i}}\left|A\left(g g \quad(q \bar{q}) \rightarrow \Omega_{Q_{1} Q_{2} Q_{3}} \bar{Q}_{1} \bar{Q}_{2} \bar{Q}_{3}\right)\right|^{2},
\end{aligned}
$$

with,

$$
d \Pi_{4}=\frac{d^{3} P}{2 E} \frac{d^{3} q_{1}}{2 E_{q_{1}}} \frac{d^{3} q_{2}}{2 E_{q_{2}}} \frac{d^{3} q_{3}}{2 E_{q_{3}}} \delta^{4}\left(k_{1}+k_{2}-P-q_{1}-q_{2}-q_{3}\right),
$$


where $\hat{s}$ is the subprocess c.m. energy squared, and the denominator $N$ in $\frac{8}{N}$ is 64 and 9 for the $g g$ fusion subprocess and the $q \bar{q}$ annihilation one, respectively, and the numerator 8 in $\frac{8}{N}$ means the summation of the spins of the heavy antiquarks in the final states, which are given randomly in the code. The spins of the initial gluons (light quark and antiquark) are given randomly in the code too.

\section{Numerical results and conclusions}

Incorporating the parton model with the cross sections of the subprocesses, the cross sections of the process $p p \rightarrow \Omega_{Q_{1} Q_{2} Q_{3}} \bar{Q}_{1} \bar{Q}_{2} \bar{Q}_{3}+X$ in $p p$ collisions can be calculated. It follows that:

$$
\begin{aligned}
\sigma & =\int d x_{1} d x_{2} f_{g_{1}}\left(x_{1}, \mu_{F}\right) f_{g_{2}}\left(x_{2}, \mu_{F}\right) \int d \hat{\sigma}\left(g g \rightarrow \Omega_{Q_{1} Q_{2} Q_{3}} \bar{Q}_{1} \bar{Q}_{2} \bar{Q}_{3}, \mu_{F}\right) \\
& +\sum_{q} \int d x_{1} d x_{2} f_{q}\left(x_{1}, \mu_{F}\right) f_{\bar{q}}\left(x_{2}, \mu_{F}\right) \int d \hat{\sigma}\left(q \bar{q} \rightarrow \Omega_{Q_{1} Q_{2} Q_{3}} \bar{Q}_{1} \bar{Q}_{2} \bar{Q}_{3}, \mu_{F}\right),
\end{aligned}
$$

where $f_{g}\left(x, \mu_{F}\right)$ and $f_{q}\left(x, \mu_{F}\right)\left(f_{\bar{q}}\left(x, \mu_{F}\right)\right)$ are the parton distribution functions of the gluon and quark (antiquark) in the proton, respectively, $\mu_{F}$ is the factorization energy scale, $\sum_{q}$ means summation of all light flavors of $q$. It happens that the predicted cross sections in the leading order are just the same for the production of the baryons consisting of identical fermions and non-identical ones. It is no longer valid beyond the leading order.

Given the mass parameters $m_{c}=1.5 \mathrm{GeV}$ and $m_{b}=4.9 \mathrm{GeV}$, the values of $|\Psi(0,0)|$ 's can be evaluated by the formula given in Ref. [6]. They are $0.0781 \mathrm{GeV}^{3}$, $0.0864 \mathrm{GeV}^{3}$, for the $\Omega_{c c c}$ and $\Omega_{c c b}\left(\Omega_{c c b}^{*}\right)$ states, respectively.

Our numerical results show that the contributions to the total cross sections from the $g g$ fusion subprocesses dominate as expected. In fact, contributions from the $g g$ fusion subprocesses are 2 3 orders of magnitude higher than that from the $q \bar{q}$ annihilation subprocesses. Thus contributions to the cross sections from the $q-\bar{q}$ annihilation subprocesses are negligible.

To carry our numerical calculations we take Cteq61 [18] parton distribution functions. Some uncertainties arise from the ambiguities in choosing the factorization energy scale $\mu_{F}$. For comparison, here we take two different values of $\mu_{F}$, i.e., $\mu_{F}=\mu_{R} / 2$ and $\mu_{F}=\mu_{R}$, where $\mu_{R}^{2}=P_{T}^{2}+\bar{M}^{2}$. 
Table 1: Predicted cross sections (in unit $n b$ ) of the triply heavy baryon production at LHC with $\sqrt{S}=7 \mathrm{TeV}$. Three typical $P_{T}$ cuts are adopted. Pseudo-rapidity cuts $|\eta|<2.5$ for CMS and ATLAS, and $1.9<\eta<4.9$ for LHCb are taken.

\begin{tabular}{|c|c|c|c|c|c|}
\hline \hline- & - & \multicolumn{2}{|c|}{ LHC (CMS, ATLAS $)$} & \multicolumn{2}{c|}{ LHCb } \\
\hline- & \multicolumn{2}{|c|}{$\eta_{c u t}$} & \multicolumn{2}{|c|}{$|\eta|<2.5$} & \multicolumn{2}{c|}{$1.9<\eta<4.9$} \\
\hline$\mu_{F}$ & - & $\mu_{R}$ & $\mu_{R} / 2$ & $\mu_{R}$ & $\mu_{R} / 2$ \\
\hline$\Omega_{c c c}$ & $0 \mathrm{GeV}$ & 0.0604 & 0.132 & 0.0329 & 0.0724 \\
- & $5 \mathrm{GeV}$ & 0.00599 & 0.0140 & 0.00163 & 0.00391 \\
- & $10 \mathrm{GeV}$ & $2.6 \mathrm{E}-4$ & $6.3 \mathrm{E}-4$ & $4.8 \mathrm{E}-5$ & $1.21 \mathrm{E}-4$ \\
\hline$\Omega_{c c b}^{*}$ & $0 \mathrm{GeV}$ & 0.00151 & 0.00351 & $7.24 \mathrm{E}-4$ & 0.00172 \\
- & $5 \mathrm{GeV}$ & $6.49 \mathrm{E}-4$ & 0.00152 & $1.89 \mathrm{E}-4$ & $4.54 \mathrm{E}-4$ \\
- & $10 \mathrm{GeV}$ & $9.62 \mathrm{E}-5$ & $2.26 \mathrm{E}-4$ & $1.95 \mathrm{E}-5$ & $4.67 \mathrm{E}-5$ \\
\hline$\Omega_{c c b}$ & $0 \mathrm{GeV}$ & $4.89 \mathrm{E}-4$ & 0.00114 & $2.15 \mathrm{E}-4$ & $5.09 \mathrm{E}-4$ \\
- & $5 \mathrm{GeV}$ & $2.43 \mathrm{E}-4$ & $5.67 \mathrm{E}-4$ & $6.86 \mathrm{E}-5$ & $1.65 \mathrm{E}-4$ \\
- & $10 \mathrm{GeV}$ & $4.49 \mathrm{E}-5$ & $1.05 \mathrm{E}-4$ & $0.894 \mathrm{E}-5$ & $2.13 \mathrm{E}-5$ \\
\hline
\end{tabular}

We calculate the production cross sections of the baryons $\Omega_{c c c}, \Omega_{c c b}^{*}$ and $\Omega_{c c b}$ at LHC with total energy $\sqrt{S}=7 \mathrm{TeV}$ and $\sqrt{S}=14 \mathrm{TeV}$ and list in Table 1 and Table 2 with various $P_{T}$ and pseudo-rapidity cuts, respectively. From the tables, we see that the predicted numerical results differ by around factor 2 with two different factorization scales $\mu_{F}=\mu_{R} / 2$ and $\mu_{F}=\mu_{R}$. These uncertainties are expected to be reduced by including the next leading calculations which will be extremely difficult work.

From Table 1, we see that for $10 \mathrm{fb}^{-1}$ integrated luminosity running at $7 \mathrm{TeV}$, for the production of $\Omega_{c c c}$, around $(0.6-1.4) \times 10^{5}$ events at CMS and ATLAS can be accumulated with kinematic cuts $P_{T}>5 \mathrm{GeV}$ and $|\eta|<2.5$, while this number is around $(2-4) \times 10^{4}$ with kinematic cuts $P_{T}>5 \mathrm{GeV}$ and $1.9<\eta<4.9$ at LHCb. For the production of $\Omega_{c c b}$, both $\Omega_{c c b}$ and $\Omega_{c c b}^{*}$ needs to add together since the higher mass state will decay into the lower mass state by emitting a photon. After doing this, we see that for $10 \mathrm{fb}^{-1}$ integrated luminosity running at $7 \mathrm{TeV}$, for the production of $\Omega_{c c b}$, around $(1-2) \times 10^{4}$ events at CMS and ATLAS can be accumulated with 
Table 2: Predicted cross sections (in unit $n b$ ) of the triply heavy baryon production at LHC with $\sqrt{S}=14 \mathrm{TeV}$. Three typical $P_{T}$ cuts are adopted. Pseudo-rapidity cuts $|\eta|<2.5$ for CMS and ATLAS, and $1.9<\eta<4.9$ for LHCb are taken.

\begin{tabular}{|c|c|c|c|c|c|}
\hline \hline- & - & \multicolumn{2}{|c|}{ LHC (CMS, ATLAS) } & \multicolumn{2}{c|}{ LHCb } \\
\hline- & \multicolumn{2}{|c|}{$\eta_{c u t}$} & \multicolumn{2}{|c|}{$|\eta|<2.5$} & \multicolumn{2}{c|}{$1.9<\eta<4.9$} \\
\hline$\mu_{F}$ & - & $\mu_{R}$ & $\mu_{R} / 2$ & $\mu_{R}$ & $\mu_{R} / 2$ \\
\hline$\Omega_{c c c}$ & $0 \mathrm{GeV}$ & 0.113 & 0.216 & 0.0684 & 0.135 \\
- & $5 \mathrm{GeV}$ & 0.0123 & 0.0258 & 0.00412 & 0.00906 \\
- & $10 \mathrm{GeV}$ & 0.000625 & 0.00136 & 0.000145 & 0.000349 \\
\hline$\Omega_{c c b}^{*}$ & $0 \mathrm{GeV}$ & 0.00320 & 0.00677 & 0.00175 & 0.00378 \\
- & $5 \mathrm{GeV}$ & 0.00143 & 0.00307 & 0.000521 & 0.00114 \\
- & $10 \mathrm{GeV}$ & $2.34 \mathrm{E}-4$ & $5.03 \mathrm{E}-4$ & $0.625 \mathrm{E}-4$ & $1.38 \mathrm{E}-4$ \\
\hline$\Omega_{c c b}$ & $0 \mathrm{GeV}$ & 0.00105 & 0.00222 & 0.000527 & 0.00115 \\
- & $5 \mathrm{GeV}$ & 0.000544 & 0.00117 & 0.000190 & 0.000419 \\
- & $10 \mathrm{GeV}$ & 0.000109 & 0.000236 & $0.289 \mathrm{E}-4$ & $0.639 \mathrm{E}-4$ \\
\hline
\end{tabular}


kinematic cuts $P_{T}>5 \mathrm{GeV}$ and $|\eta|<2.5$, while this number is around $(2-6) \times 10^{3}$ with kinematic cuts $P_{T}>5 \mathrm{GeV}$ and $1.9<\eta<4.9$ at $\mathrm{LHCb}$.

From Table 2, we see that for $1000 \mathrm{fb}^{-1}$ integrated luminosity running at $14 \mathrm{TeV}$, for the production of $\Omega_{c c c}$, around $(0.6-1.4) \times 10^{6}$ events at CMS and ATLAS can be accumulated with kinematic cuts $P_{T}>10 \mathrm{GeV}$ and $|\eta|<2.5$, while this number is around $(4-9) \times 10^{6}$ with kinematic cuts $P_{T}>5 \mathrm{GeV}$ and $1.9<\eta<4.9$ at LHCb. For the production of $\Omega_{c c b}$, again by adding both $\Omega_{c c b}$ and $\Omega_{c c b}^{*}$ events together, we see that for $1000 \mathrm{fb}^{-1}$ integrated luminosity running at $14 \mathrm{TeV}$, for the production of $\Omega_{c c b}$, around $(3-7) \times 10^{5}$ events at CMS and ATLAS can be accumulated with kinematic cuts $P_{T}>10 \mathrm{GeV}$ and $|\eta|<2.5$ while this number is around $(0.7-1.5) \times 10^{6}$ with kinematic cuts $P_{T}>5 \mathrm{GeV}$ and $1.9<\eta<4.9$ at LHCb.

Here we calculate only the contributions from the ground states. Excited states also can decay into the ground states. Including those contributions, the real production rates should be $2-3$ times higher than ones listed in Table 1 and Table 2 . Moreover, the cross sections for the anti-triply heavy baryons are exactly the same. This will double the accumulated triply heavy baryon events. From the tables, we see that the cross sections for LHC running at $14 \mathrm{TeV}$ is about 2 times larger than that for LHC running at $7 \mathrm{TeV}$.

We show the $P_{T}$-distributions and the rapidity distributions of the productions of the triply heavy baryons in Figs. $2-7$ at LHC with various LHC running energies and in various detectors. The differential cross section drops fast with increasing $P_{T}$ as expected. Some of the parts in the $P_{T}$ distribution are not smooth due to Vegas simulation error which can be reduced by increasing the event numbers.

It is instructive to look at the fragmentation description for the production of the triply heavy baryons. One expects that at large $P_{T}$ the production is dominated by the fragmentation mechanism. The production cross sections are then factored into the product of the cross sections of the heavy quark pair production and the fragmentation function. As dimensionless quantities, the QCD leading order fragmentation function and the fragmentation probability must be proportional to $\alpha_{s}^{4}|\Psi(0,0)|^{2} / m^{6}$. Since the process is complicated, one cannot expect to have a simpler analytic expression.

Comparing our predicted results for the $\Omega_{c c c}$ production with those fragmentation model descriptions presented in [12, 13], we see that our numerical result is around 2-3 
orders of magnitude higher than that given in [12] and around 2 orders of magnitude smaller than that given in [13] for $P_{T}>10 \mathrm{GeV}$. These large discrepancies cannot be reconciled by varying parameters such as the heavy quark mass, factorization energy scale, type of the parton distribution function, and the rapidity cuts used in the calculations.

To look for the reasons leading to so huge discrepancies between our result and those in [12, 13], we first look at the nonperturbative parameter used in the calculations. In [13], the authors introduced a nonperturbative dimension-one parameter $f_{B}$ as used in the meson production when they calculated the amplitude of the production of the triply heavy baryon. From Eq.(5) in Ref. [13, the nonperturbative parameter $f_{B}$ they introduced can be related to our $\Psi_{c c c}(0,0)$ by $\left|\Psi_{c c c}(0,0)\right|^{2} \sim f_{B}^{2} \cdot M_{\Omega} m_{c}^{3}$. In their calculation, they took $f_{B}^{2}=0.0625 \mathrm{GeV}^{2}$ corresponding to a value of the $\left|\Psi_{c c c}(0,0)\right|^{2}$ in our paper being $0.458 \mathrm{GeV}^{6}$, which is around two orders of magnitude higher than the value we used. From quark model calculations, this value is certainly over-estimated. This may explain the two orders of magnitude discrepancy between the results in [13] and ours.

In [12], the author introduced two nonperturbative parameters $\psi_{c c}(0)$ and $\psi_{c c c}^{\prime}(0)$ describing the wave function at origin of the double quarks forming the diquark and the quark and the diquark forming the baryon, respectively. His fragmentation function was then proportional to $\left|\psi_{c c}(0) \cdot \psi_{c c c}^{\prime}(0)\right|^{2}=0.00345 \mathrm{GeV}^{6}$, which was comparable to the nonperturbative parameter we used in this paper $\left|\Psi_{c c c}(0,0)\right|^{2}=0.0061 \mathrm{GeV}^{6}$. Thus the discrepancy between the results in [12] and ours cannot be attributed to the nonperturbative parameters used in the calculations. Notice that the fragmentation probability given in Eq.(8) in [12] is proportional to a number $A_{c}$ he introduced. In the following equation after Eq.(8) in [12, the author showed that $A_{c}$ was a small number $4.2 \times 10^{-3}$ which arose from a cancelation between two very large numbers (Surprisingly it is a 10-digit cancelation!). This may give explanation for the discrepancy between our results and those presented in [12]. Physically, when they calculated the fragmentation function using the diquark model, probably only tiny contributions were accounted for.

We also calculate the production cross sections for the triply heavy baryons at Tevatron. Our calculations show that there are around 2 thousands $\Omega_{c c c}$ events and 
around 3 hundreds $\Omega_{c c b}$ events accumulated with $5 \mathrm{GeV} P_{T}$-cut and 0.6 rapidity cuts for $2 \mathrm{fb}^{-1}$ integrated luminosity at Tevatron. Considering the detection difficulty, we think these numbers are not enough to discover the triply heavy baryons at Tevatron.

\section{Signatures and summary}

The particular signatures of the triply heavy baryons in the detectors can be used to reconstruct the triply heavy baryons. The ground states of the triply heavy baryons can decay only through the weak interaction. Naively, the decay width for $\Omega_{c c c}$ is triple the width of the free $c$ quark while that for $\Omega_{c c b}$ is double the width of the free $c$ quark plus the decay width of the free $b$ quark. However, one expects that it receives large corrections both from the destructive interference between identical fermions and from large recoil effects. So the total decay widths may be deviated largely from the naive estimation.

For $\Omega_{c c c}$, a very interesting non-leptonic decay mode is that:

$$
\begin{aligned}
& \Omega_{c c c} \\
& \downarrow \\
& \Omega_{c c s}+\pi^{+} \\
& \downarrow \\
& \Omega_{c s s}+\pi^{+} \\
& \downarrow \\
& \Omega_{s s s}+\pi^{+} \\
& \hline \Omega_{c c c} \rightarrow \Omega_{s s s}+3 \pi^{+} .
\end{aligned}
$$

Thus with this cascade decay mode, the $\Omega_{c c c}$ finally decays to $\Omega_{s s s}+3 \pi^{+}$, which are stable charged particles in the detectors. Combining the tracks of the charged particles and the invariant mass, the triply heavy baryons can easily be reconstructed.

For $\Omega_{c c b}$, the similar but complicated interesting non-leptonic decay mode is that: 


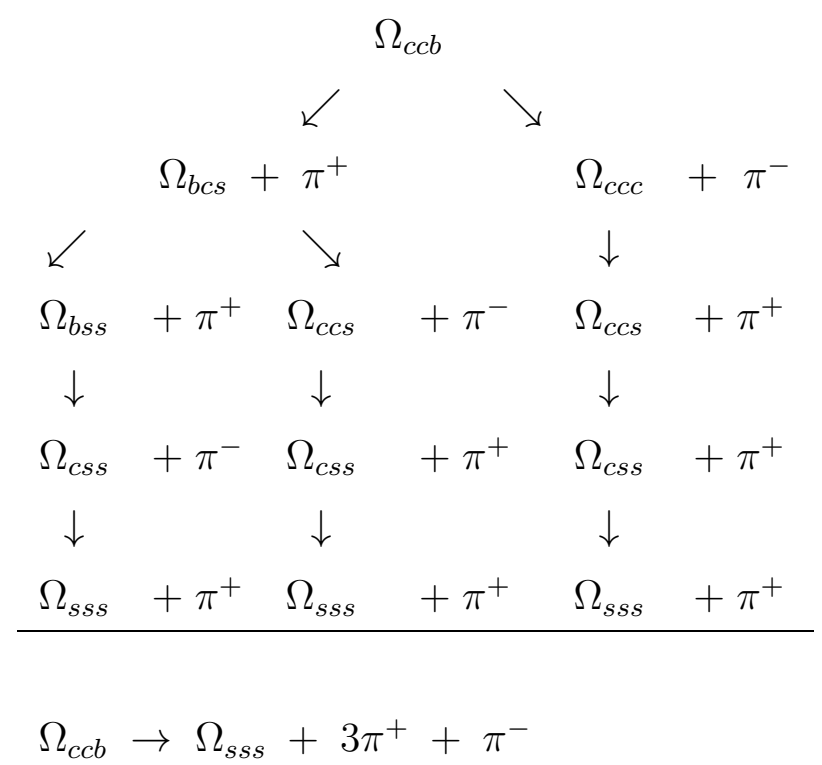

Thus with these cascade decay modes, the $\Omega_{c c b}$ final decay products are $\Omega_{s s s}+$ $3 \pi^{+}+\pi^{-}$, which are stable charged particles in the detectors. Again combining the tracks of the charged particles and the invariant mass, the triply heavy baryons can easily be reconstructed.

We can also analyze the semileptonic decays of the $\Omega_{c c c}, \Omega_{c c b}$. The cascade decays are $c \rightarrow s$ or $b \rightarrow c \rightarrow s$, with emitting leptons and neutrinos. This cascade decay feature with the decay vertex can also be used to identify the triply heavy baryons. Moreover, one can also combine the semileptonic decay with the nonleptonic decay to reconstruct the triply heavy baryons.

In conclusion, our results show that a number of triply heavy baryons events can be accumulated at LHC. They can be reconstructed with their unique signatures in detectors. Triply heavy baryons are very interesting hadrons to be explored for they provide particular information about strong interaction, hadron structure, and weak decay of heavy baryons. They are still absent in the particle data booklet after the heavy quarkonium has been discovered over three decades. Our results show that it is quite promising to discover those triply heavy baryons in LHC experiments both for large number events and for their unique signatures in detectors. One may be waiting for an exciting time to discover them at LHC. 

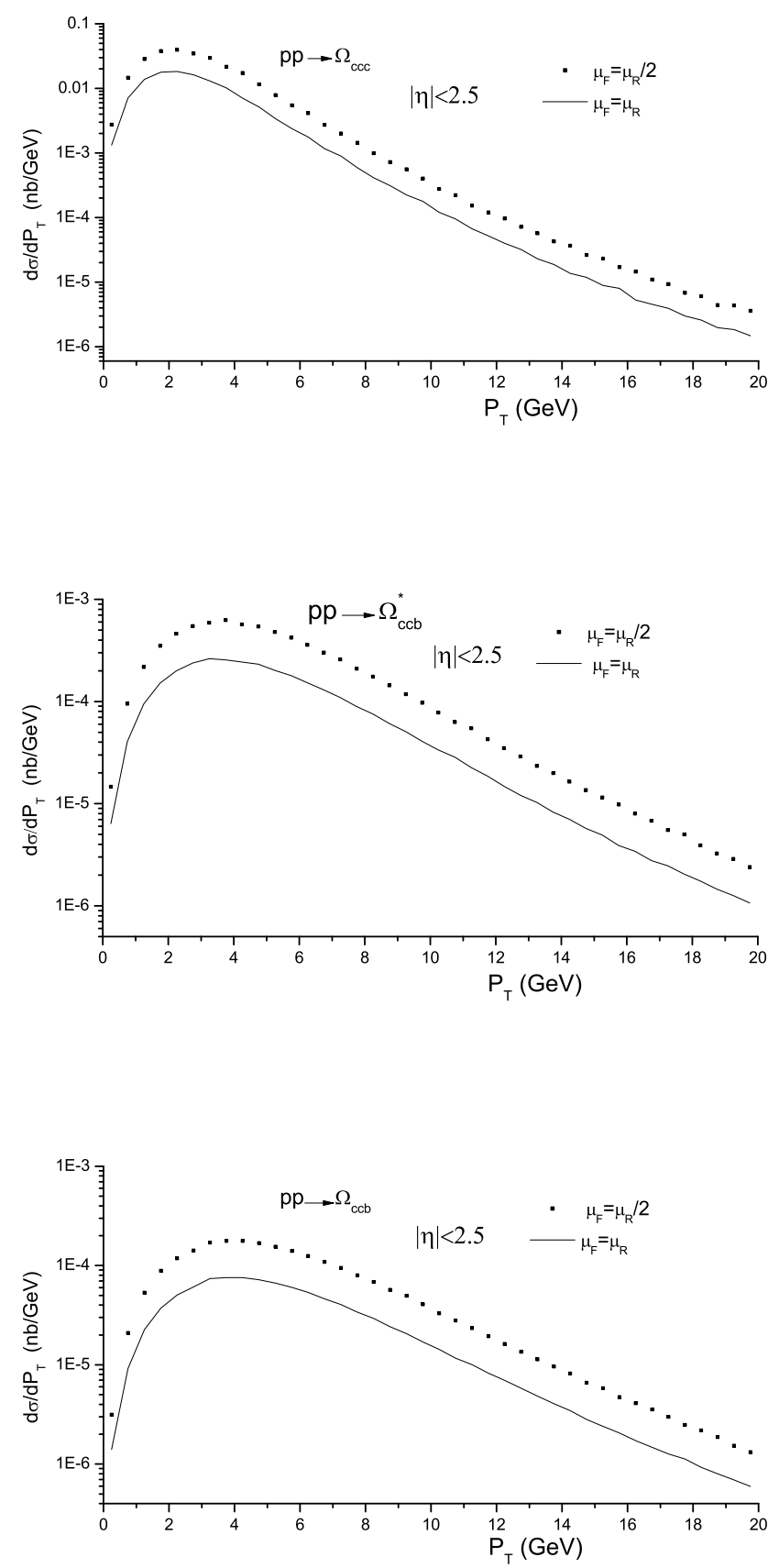

Figure 2: The predicted $P_{T}$-distributions of the triply heavy baryon production in CMS and ATLAS, with $\sqrt{S}=7 \mathrm{TeV}$. 

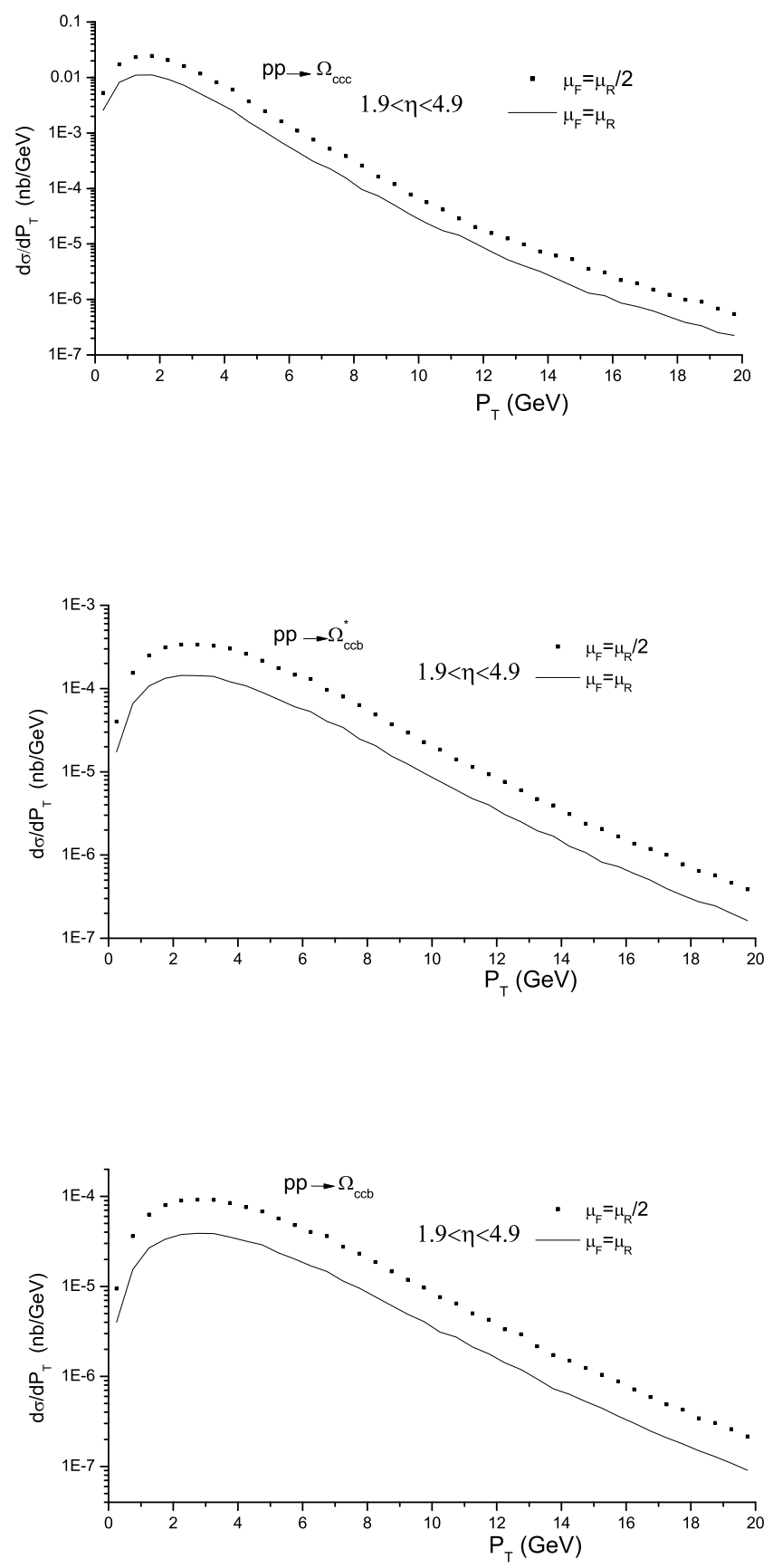

Figure 3: The predicted $P_{T^{-}}$distributions of the triply heavy baryon production in LHCb with $\sqrt{S}=7 \mathrm{TeV}$. 

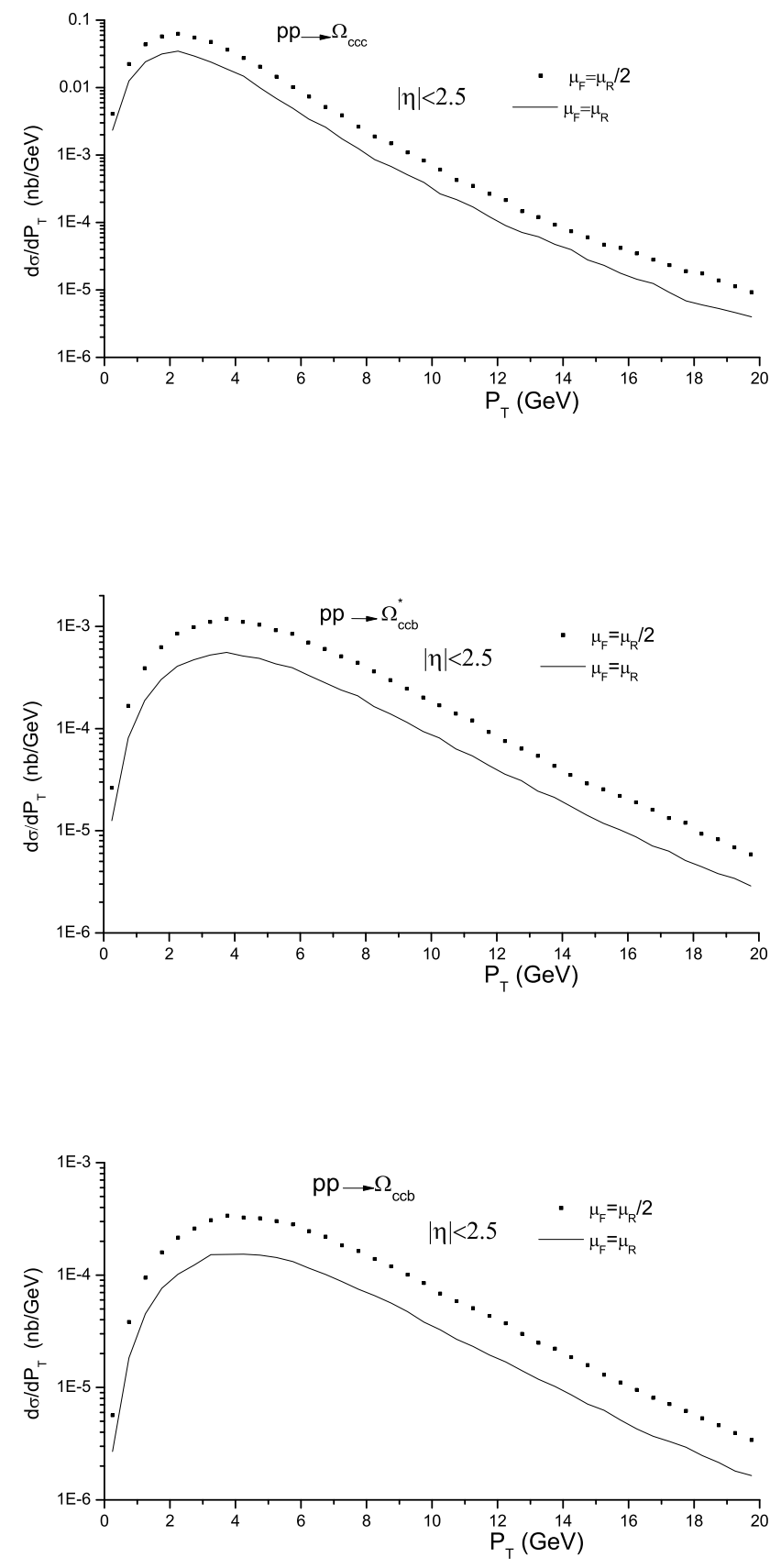

Figure 4: The predicted $P_{T}$-distributions of the triply heavy baryon production in CMS and ATLAS, with $\sqrt{S}=14 \mathrm{TeV}$. 

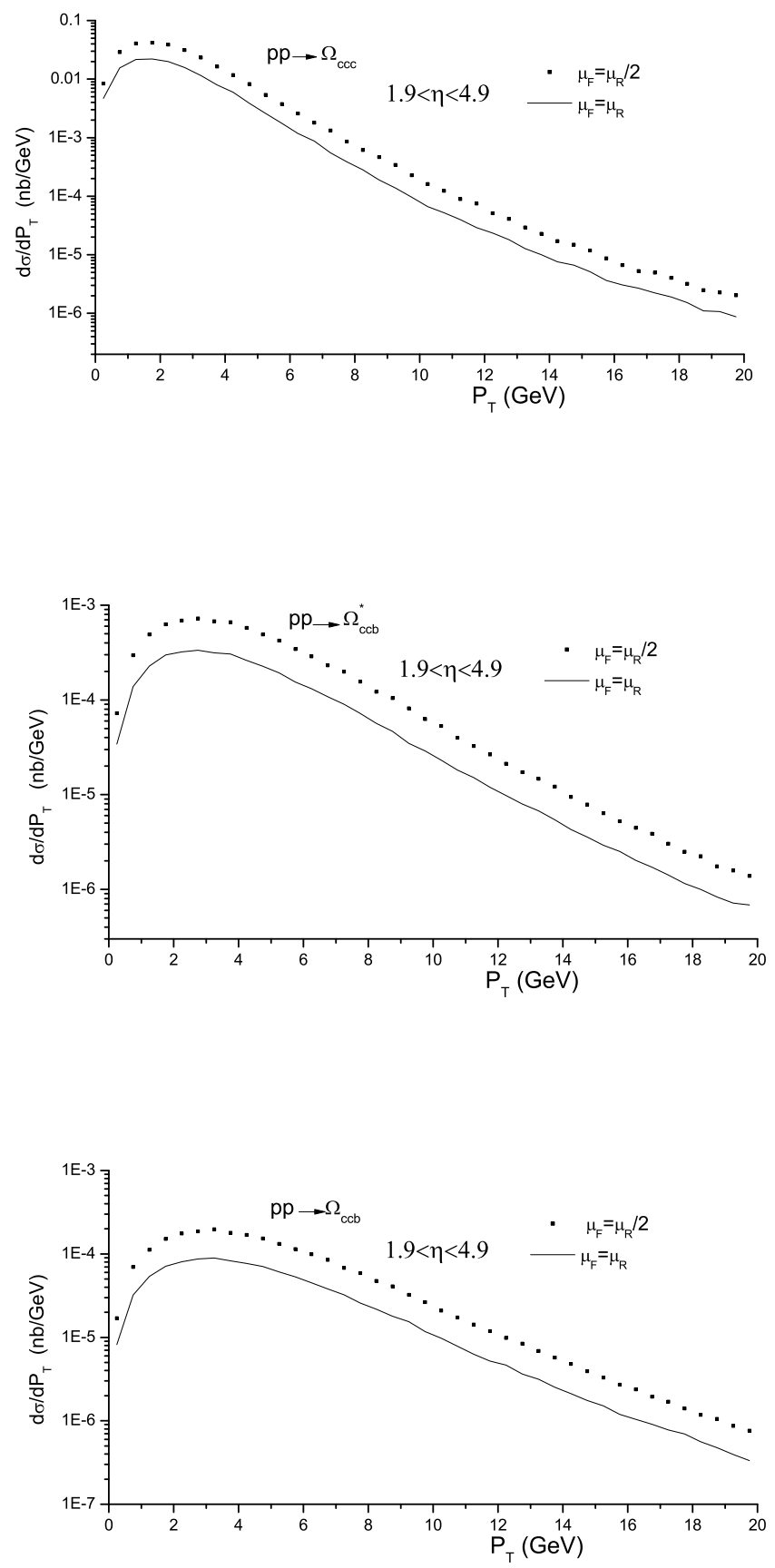

Figure 5: The predicted $P_{T}$-distributions of the triply heavy baryon production in LHCb, with $\sqrt{S}=14 \mathrm{TeV}$. 

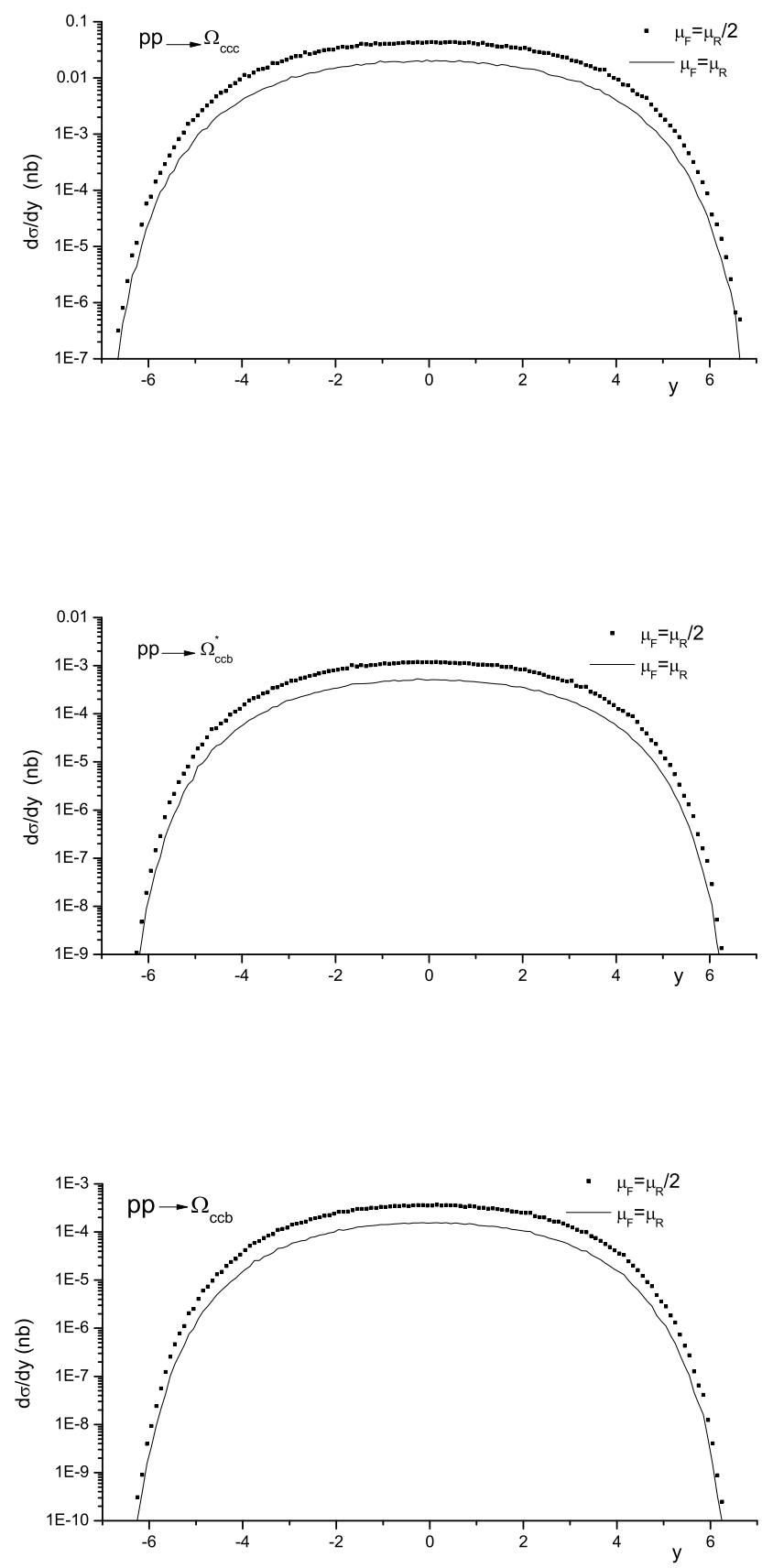

Figure 6: The predicted rapidity distributions of the triply heavy baryon production at $\mathrm{LHC}$ with $\sqrt{S}=7 \mathrm{TeV}$. 

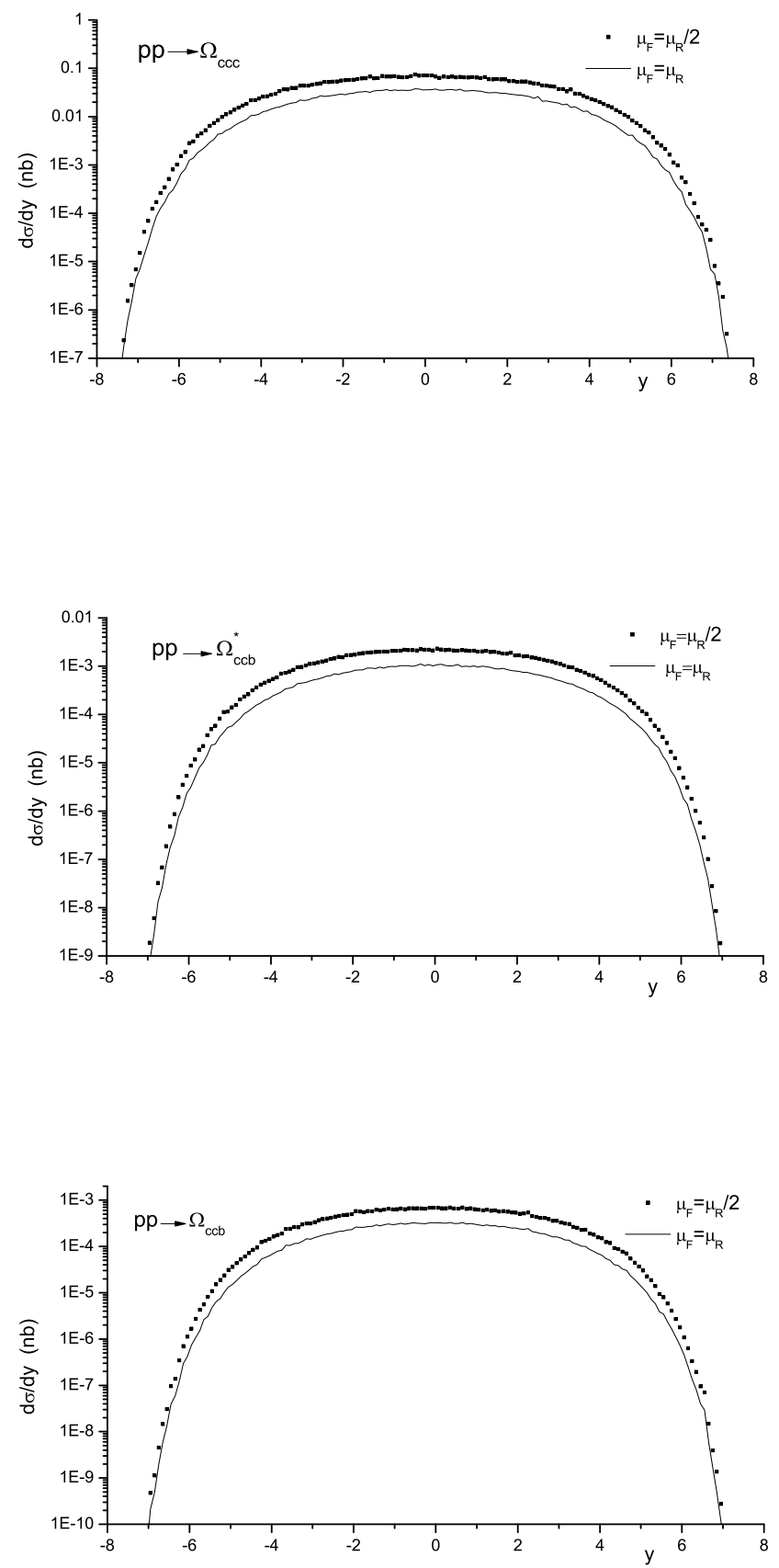

Figure 7: The predicted rapidity distributions of the triply heavy baryon production at LHC with $\sqrt{S}=14 \mathrm{TeV}$. 


\section{Acknowledgements}

One of the authors (Su-Zhi Wu) wants to thank Bin Gong for great help for matching the color factors and helpful discussions. This work is partly supported by the NSFC with contract number 10875156. 


\section{Appendix}

\subsection{Nonrelativistic states}

The $S$ - wave state of the nonrelativistic triply heavy baryon in its rest frame is constructed as:

$$
\begin{aligned}
\left|\Omega_{Q_{1} Q_{2} Q_{3}}, s, s_{Z}\right\rangle= & \sqrt{2 \bar{M}} \int \frac{d^{3} \vec{V}_{1}}{(2 \pi)^{3}} \frac{d^{3} \vec{V}_{2}}{(2 \pi)^{3}} \sum_{\xi_{1}, \xi_{2}, \xi_{3}} \sum_{\eta_{1}, \eta_{2}, \eta_{3}} \frac{\varepsilon^{\xi_{1} \xi_{2} \xi_{3}}}{\sqrt{6}}\left\langle s, s_{Z} \mid \eta_{1}, \eta_{2}, \eta_{3}\right\rangle \\
& \frac{1}{\sqrt{2 E_{1} 2 E_{2} 2 E_{3}}} \frac{1}{\sqrt{d !}} \psi\left(\vec{V}_{1}, \vec{V}_{2}\right) \times\left|Q_{1}, \xi_{1}, \eta_{1}, \vec{p}_{1}=\vec{V}_{1}\right\rangle \\
& \times\left|Q_{2}, \xi_{2}, \eta_{2}, \vec{p}_{2}=\vec{V}_{2}\right\rangle \times\left|Q_{3}, \xi_{3}, \eta_{3}, \vec{p}_{3}=-\vec{V}_{1}-\vec{V}_{2}\right\rangle,
\end{aligned}
$$

where, $\xi_{i}, \eta_{i}, m_{i}$, and $\left(E_{i}, \vec{p}_{i}\right)(i=1,2,3)$ are the color, the spin, the mass, and the four-momentum of the heavy quark $Q_{i} ; V_{1}$ and $V_{2}$ are the relative momenta among the heavy quarks; $\left\langle s, s_{Z} \mid \eta_{1}, \eta_{2}, \eta_{3}\right\rangle$ is the $\mathrm{C}-\mathrm{G}$ coefficient; $s$ and $s_{Z}$ are the spin of the baryon and its third component, respectively; $\psi\left(\vec{V}_{1}, \vec{V}_{2}\right)$ is the wave function of the baryon in the momentum space.

The quark states are normalized by:

$$
\left\langle f^{\prime}, \xi^{\prime}, \eta^{\prime}, \vec{p} \mid f, \xi, \eta, \vec{p}\right\rangle=\delta_{f^{\prime} f^{\prime}} \delta_{\xi^{\prime} \xi} \delta_{\eta^{\prime} \eta}(2 \pi)^{3} 2 E_{f} \delta^{(3)}(\vec{p}-\vec{p})
$$

where $f^{\prime}$ and $f$ are the heavy flavors.

$\psi\left(\vec{V}_{1}, \vec{V}_{2}\right)$ is normalized by:

$$
\int \frac{d^{3} \vec{V}_{1}}{(2 \pi)^{3}} \frac{d^{3} \vec{V}_{2}}{(2 \pi)^{3}} \psi^{*}\left(\vec{V}_{1}, \vec{V}_{2}\right) \psi\left(\vec{V}_{1}, \vec{V}_{2}\right)=1 .
$$

By the Eqs. (12) and (13), we know the baryon is normalized as:

$$
\left\langle\Omega, s^{\prime}, s_{Z}^{\prime}, \vec{P}^{\prime} \mid \Omega, s, s_{Z}, \vec{P}\right\rangle=(2 \pi)^{3} 2 E \delta^{s, s^{\prime}} \delta^{s_{Z}, s_{Z}^{\prime}} \delta^{(3)}\left(\vec{P}^{\prime}-\vec{P}\right) .
$$

The wave function at the origin is related to $\psi\left(\vec{V}_{1}, \vec{V}_{2}\right)$ by:

$$
\Psi(0,0)=\int \frac{d^{3} \vec{V}_{1}}{(2 \pi)^{3}} \frac{d^{3} \vec{V}_{2}}{(2 \pi)^{3}} \psi\left(\vec{V}_{1}, \vec{V}_{2}\right) .
$$




\subsection{Spinor and polarization vector}

For on-shell quark or antiquark with 4-momentum $p^{\mu}=\left(p^{0}, p^{1}, p^{2}, p^{3}\right)$ and mass $m$ satisfying $p^{2}=m^{2}$, the spinor of the quark reads:

$$
\begin{aligned}
u^{\frac{1}{2}}(p) & =\left(f_{1}\left(|\vec{p}|+p^{3}, p^{1}+i p^{2}\right), f_{2}\left(|\vec{p}|+p^{3}, p^{1}+i p^{2}\right)\right), \\
u^{-\frac{1}{2}}(p) & =\left(f_{2}\left(-p^{1}+i p^{2},|\vec{p}|+p^{3}\right), f_{1}\left(-p^{1}+i p^{2},|\vec{p}|+p^{3}\right)\right),
\end{aligned}
$$

and the spinor of the antiquark reads:

$$
\begin{aligned}
v^{\frac{1}{2}}(p) & =\left(-f_{2}\left(-p^{1}+i p^{2},|\vec{p}|+p^{3}\right), f_{1}\left(-p^{1}+i p^{2},|\vec{p}|+p^{3}\right)\right), \\
v^{-\frac{1}{2}}(p) & =\left(f_{1}\left(|\vec{p}|+p^{3}, p^{1}+i p^{2}\right),-f_{2}\left(|\vec{p}|+p^{3}, p^{1}+i p^{2}\right)\right),
\end{aligned}
$$

where

$$
f_{1}=\frac{\sqrt{p^{0}-|\vec{p}|}}{\sqrt{2|\vec{p}|\left(|\vec{p}|+p^{3}\right)}}, \quad f_{2}=\frac{\sqrt{p^{0}+|\vec{p}|}}{\sqrt{2|\vec{p}|\left(|\vec{p}|+p^{3}\right)}} .
$$

Setting the beam line as the Z-axis, two physical polarization vectors of the gluon are:

$$
\epsilon_{\mu}^{+}=(0,1,0,0), \quad \epsilon_{\mu}^{-}=(0,0,1,0) .
$$

\subsection{The $\lambda$ matrices and the Feynman rules}

In our calculations, the $\lambda$ matrices of $S U\left(N_{c}\right)$ satisfy:

$$
\begin{aligned}
& {\left[\lambda^{a}, \lambda^{b}\right]=i f^{a b c} \lambda^{c}, \quad \operatorname{tr}\left[\lambda^{a}, \lambda^{b}\right]=\delta^{a b},} \\
& \sum_{a}\left(\lambda^{a}\right)_{i j}\left(\lambda^{a}\right)_{k l}=\delta_{i l} \delta_{j k}-\frac{1}{N_{c}} \delta_{i j} \delta_{k l},
\end{aligned}
$$

where $N_{c}=3$ in QCD.

Feynman rules for those vertex are given as follows.

Quark-antiquark-gluon vertex:

$$
i \frac{g}{\sqrt{2}} \gamma_{\mu}\left(\lambda^{a}\right)_{i j}
$$


Tri-Vector vertex in order (123) with all momenta incoming to vertex:

$$
\frac{g}{\sqrt{2}} f^{a_{1} a_{2} a_{3}}\left[\left(p_{1}-p_{2}\right)_{\mu_{3}} g_{\mu_{1} \mu_{2}}+\left(p_{2}-p_{3}\right)_{\mu_{1}} g_{\mu_{2} \mu_{3}}+\left(p_{3}-p_{1}\right)_{\mu_{2}} g_{\mu_{1} \mu_{3}}\right]
$$

Quadruple-Vector vertex:

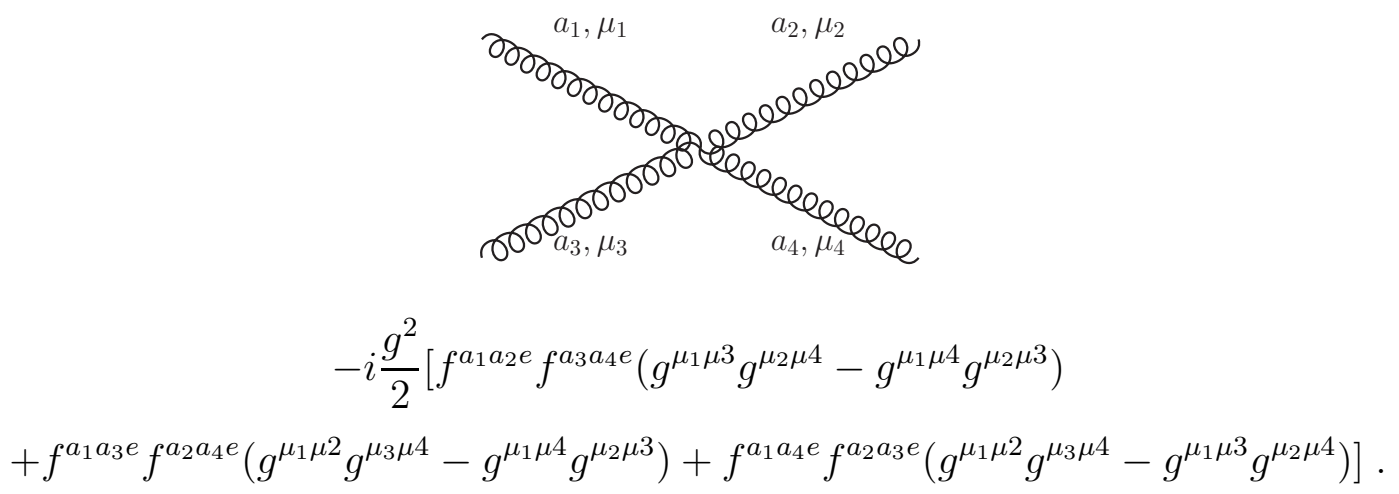

\section{References}

[1] G. T. Bodwin, E. Braaten, and G. P. Lepage, Phys. Rev. D 51, 1125 (1995), Erratum-ibid. D 55, 5853 (1997).

[2] Edward Witten, Nucl. Phys. B 160, 57 (1979).

[3] P. Hasenfratz, R. R. Horgan, J. Kuti and J. M. Richard, Phys. Lett. B 94, 401 (1980); J.-P. Ader, J.-M. Richard and P. Taxil, Phys. Rev. D 25, 2370 (1982).

[4] Masayasu Tsuge, Toshiyuki Morii and Jun-ya Morishita, Mod. Phys. Lett. A 1, 131 (1986), Erratum-ibid. A 2, 283 (1987); Francesco Becattini, Phys. Rev. Lett. 95, 022301 (2005).

[5] A. P. Martynenko, Phys. Lett. B 663, 317 (2008).

[6] Yu Jia, JHEP 0610 (2006) 073.

[7] Stefan Meinel, arXiv:1008.3154v2 [hep-lat].

[8] J. D. Bjorken, in Proceedings of the International Conference on Hadron Spetroscopy, College Park, MD, 1985 (FERMILAB-CONF-85-069). 
[9] Nora Brambilla and Antonio Vairo, Phys. Rev. D 72, 034021 (2005); Antonio Vairo, arXiv:1008.4473v2 (2010).

[10] Jian-Rong Zhang, Ming-Qiu Huang, Phys. Lett. B 674, 28 (2009); Bhavin Patel, Ajay Majethiya, P. C. Vinodkumar, arXiv:0808.2880 [hep-ph].

[11] S. P. Baranov and V. L. Slad, Phys. Atom. Nucl. 67, 808 (2004).

[12] V. A. Saleev, Mod. Phys. Lett. A 14 (1999) 2615 arXiv:hep-ph/9906515].

[13] M. A. Gomshi Nobary and R. Sepahvand, Phys. Rev. D71, 034024 (2005); Nucl. Phys. B 741, 34 (2006); M. A. Gomshi Nobary, Phys. Lett. B 559, 239 (2003), Erratum-ibid. B 598, 294 (2004).

[14] Y. Q. Chen, Phys. Rev. D 48 , 5181 (1993).

[15] Chao-Hsi Chang, Yu-Qi Chen and Robert J. Oakes, Phys. Rev. D 54, 4344 (1996).

[16] C. H. Chang and Y. Q. Chen, Phys. Rev. D 46, 3845 (1992), Erratum-ibid. D 50, 6013 (1994).

[17] V. Barger, A. L. Stange and R. J. N. Phillips, Phys. Rev. D 44, 1987 (1991); Chao-Hsi Chang and Yu-Qi Chen, Phys. Rev. D 48, 4086 (1993).

[18] J. Pumplin, D. R. Stump, J. huston, H. L. Lai, P. Nadolsky, and W. K. Tung, hep-ph/0201195. 